\title{
Guerra, hambre y aventura en la vida de Cándida Sala, enfermera de Cruz Roja ${ }^{\star}$
}

\section{War, hunger and adventure in the life of Candida Sala, red Cross Nurse}

\section{Guerra, fome e aventura na vida de Cándida Sala, enfermeira da Cruz Vermellha}

Carmen Torres Penella ${ }^{1}$, Ana Ramió Jofré ${ }^{2}$, Roser Valls Molin ${ }^{3}$.

${ }^{1}$ C. Profesora Titular de Universidad. Departamento de Enfermería. Universidad de Lleida.

${ }^{2}$ Profesora Titular. Escuela de Enfermería de San Juan de Dios Barcelona.

${ }^{3}$ Catedrática de Enfermería. Escuela de Enfermería de la Universidad de Barcelona.

${ }^{1,2,3}$ Profesoras de "Historia de la Enfermería" y miembros del Grupo FEBE para el estudio y docencia de la Historia de Enfermería.

Cómo citar este artículo en edición digital: Torres, C; Ramió, A; Valls, R. . (2012) Guerra, hambre y aventura en la vida de Cándida Sala, enfermera de Cruz Roja. 16, 34. Disponible en: http://dx.doi.org/10.7184/cuid.2012.34.03>

Correspondencia: Universitat de Lleida Campus de Ciències de la Salut Av. de l'Alcalde Rovira Roure, 44 E-25198 Lleida. e-mail: carme.torres@infermeria.udl.cat<carme.torres@infermeria.udl.cat> Recibido 20/02/2012/ Aceptado: 11/05/2012

* III Simposio iberoamericano de historia de la enfermería, XII congreso nacional, VII internacional de historia de la enfermería y VII jornadas internacionales de cultura de los cuidados. Alicante 2011.

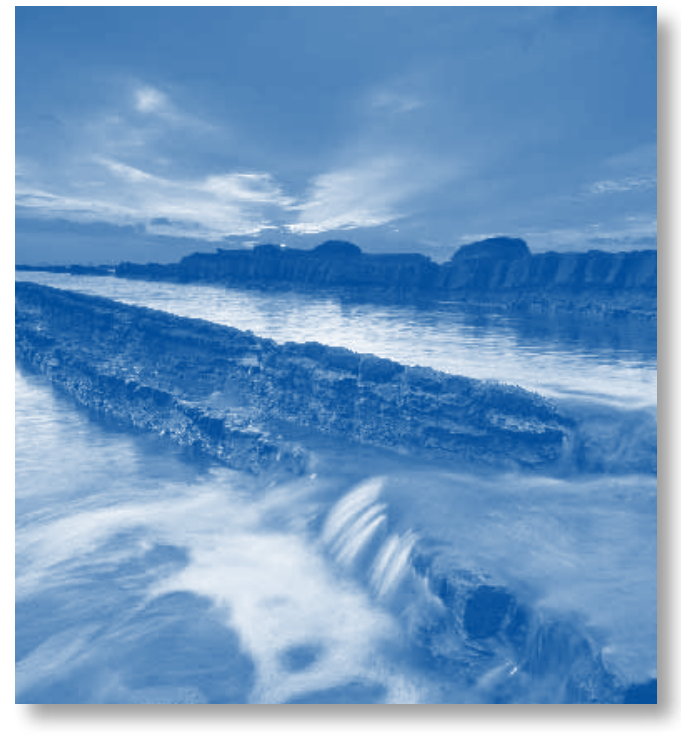

ABSTRACT

The aim of this work is to highlight the account of Cándida, a volunteer nurse in the Spanish Civil War in Catalonia, near the Segre front, with a view to understanding her nursing contribution in the war.

A qualitative historical study based on oral history, observation and analysis of personal documents, and on bibliographic texts. Content analysis and progress analysis are conducted.

Notable in her account is the traumatic impact of the war on the young woman, social and family commitment as a source of motivation for her becoming involved in the war, the humanitarian nature of the care she gave, the curative and preventive treatments that she applied to infectious and wounded patients. In the post-war period, hunger accompanied her studies as a Red Cross nurse, professional work as a nurse in various hospital centres and services in Barcelona and abroad. It concludes by stressing her qualities as a "young woman ahead of her time" showing her to be a strong woman, committed to society and family, with a rebellious and adventuring spirit, who was able to understand her professional and personal role well, fulfilling her responsibilities at all times.

Keywords: Nurses, care, Spanish Civil War, Historical Memory. 


\section{RESUMO}

O objetivo deste trabalho é dar a conhecer o testemunho de Cándida, enfermeira voluntária da Guerra Civil Espanhola no território catalão, perto da frente do rio Segre, para assim percebermos o seu papel como enfermeira no conflito bélico.

É um estudo histórico de conceição qualitativa, realizado a partir da história oral, da observação e análise de documentos pessoais e textos bibliográficos. Adicionalmente, faz-se uma análise de conteúdo e outra de progressão.

Do seu testemunho destaca-se o impacto traumático da guerra na jovem, o compromisso social e familiar como fonte de motivação para o envolvimento na guerra, o caráter humanitário dos cuidados que prestava, os tratamentos curativos e preventivos que aplicava em doentes infecciosos e feridos. Já no pós-guerra, são abordados a fome que acompanhou os estudos de enfermeira da Cruz Vermelha e o trabalho profissional de enfermeira em diversos centros e serviços hospitalares de Barcelona e no estrangeiro. Com a exaltação das qualidades de "jovem à frente do seu tempo", mostra-se uma mulher forte, comprometida com a sociedade e a sua família, de espírito rebelde e aventureiro, que soube entender bem o seu papel profissional e pessoal e assumir em cada momento as suas responsabilidades.

Palavras-chave: Enfermeiras, cuidados, Guerra Civil Espanhola, Memória Histórica.

\section{RESUMEN}

El objetivo de este trabajo es dar a conocer el testimonio de Cándida, enfermera voluntaria de la Guerra Civil Española en el territorio catalán, cerca del frente del Segre, con el fin de comprender su contribución enfermera en el conflicto bélico.
Es un estudio histórico de diseño cualitativo, a partir de la historia oral, la observación y análisis de documentos personales y textos bibliográficos. Se realiza un análisis de contenido y análisis de progreso. De su testimonio se destaca el impacto traumático de la guerra en la joven, el compromiso social y familiar como fuente de motivación para implicarse en la guerra, el carácter humanitario de los cuidados que dispensaba y los tratamientos curativos y preventivos que aplicaba a enfermos infecciosos y heridos. Ya en la posguerra, el hambre acompañó a los estudios de enfermera de Cruz Roja, el trabajo profesional de enfermera en diversos centros y servicios hospitalarios de Barcelona y en el extranjero. Se concluye, ensalzando las cualidades de "joven adelantada a su tiempo" por mostrarse como mujer fuerte, comprometida con la sociedad y su familia, de espíritu rebelde y aventurero, que supo entender bien su papel profesional y personal asumiendo en cada momento sus responsabilidades.

Palabras clave: Enfermeras, cuidados, Guerra Civil Española, Memoria Histórica.

\section{INTRODUCCIÓN Y OBJETIVOS}

Las dos últimas décadas han sido muy fructíferas en la historiografía de la enfermería en la Guerra Civil española (GCE), (Siles, 1996), (Amezcua, 1996), (Nash, 1999), (Jackson, 2004), (Diaz, 2005), (Larraz, 2005), (Via, 2006). Cataluña contribuyó en el año 2005 con un proyecto de investigación becado por la "Agència de Gestió d'Ajuts Universitaris i de Recerca" (AGAUR) para dar visibilidad a las enfermeras del territorio catalán durante la contienda bélica. Fruto de este proyecto, realizado por profesoras de enfermería de las Universidades catalanas e integrantes del gru- 
po FEBE, publicó la obra "Infermeres catalanes en la Guerra Civil espanyola” (Valls, 2008). El texto recoge el testimonio oral y directo de 10 enfermeras. Fue presentado el año 2008 en el Paraninfo de Universidad de Barcelona en presencia de las enfermeras de guerra protagonistas. Así mismo, con anterioridad se celebró un homenaje de reconocimiento a las mismas en la Universidad Ramon Llull de Barcelona.

Oficialmente los proyectos se cierran, pero el ansia de nuevos conocimientos sobre el tema, cuando se trata de la recuperación de la memoria histórica, permanece siempre abierto. La aparición de nuevos testimonios que permitan ampliar el conocimiento histórico sobre la contribución enfermera en la GCE es siempre deseada. ¿Pero es posible? ¿Se ha dicho todo sobre la enfermería catalana en la GCE? Después de 75 años, ¿queda alguna enfermera que pueda contar su experiencia?

Los objetivos de este estudio son dar a conocer el testimonio de Cándida Sala Llanes, enfermera voluntaria de guerra en el frente del Segre, con el fin de comprender su implicación en el conflicto bélico, complementando el estudio de las enfermeras catalanas y aumentar con su visión la contribución enfermera en la GCE.

\section{MATERIAL Y MÉTODO}

Se plantea un estudio histórico de diseño cualitativo, a partir de la historia oral, la observación y análisis de documentos personales y textos bibliográficos.

El método de búsqueda es el de proximidad, utilizando la técnica de "bola de nieve" a través de conocidos y entidades. Los contactos previos se hicieron por teléfono, se explicaba el motivo del interés por conocerla, el objetivo concreto del estudio, se pedía su consentimiento y se fijaba día y hora para el primer encuentro. La recogida de información relativa a sus experiencias de enfermera de guerra, se realizó a través de la entrevista biográfica (Pujadas, 1992) siguiendo un guión previamente validado por expertos. En él se contempla aspectos relacionados con la motivación, la formación de enfermera, el desarrollo de su actividad de enfermera en la guerra, sucesos de este periodo y si continuó de enfermera después de la GCE.

Los tres encuentros para la entrevista se realizaron entre abril y octubre de 2011 en el domicilio familiar. Estos se desarrollaron en un clima respetuoso, de amistad y colaboración a través de un diálogo abierto - de preguntas abiertas basado en el guión - procurando como entrevistadoras obtener respuestas lo más claras y precisas que permitiesen validarlas objetivamente con otras fuentes.

Las conversaciones se registraron con la ayuda de una grabadora digital con el programa Digital Voice. La transcripción y análisis de las mismas para la construcción del relato biográfico, se realiza adoptando las normas de transcripción del fondo "Archivos de la Memoria” (Amezcua-Hueso 2004). Se ha desestimado información que carecía de capacidad ilustrativa. El análisis del relato se sustenta en el enfoque de análisis de contenido y análisis de progreso. Finalmente se interpretan los resultados del análisis y se formulan las conclusiones (Amezcua-Hueso 2009). En las revisiones con la entrevistada y su familia se incorporaba todas aquellas modificaciones y notas adicionales que se consideraban oportunas. También se ha llevado a cabo una selección, registro y análisis de documentos personales (títulos académicos, cartas, certificados y fotografías) como evidencias históricas que dan soporte al relato. En este estudio, se implementan los criterios de rigor de la investigación cualitativa 
denominados de confiabilidad (Lincoln, Y.S. Guba, 1985).

\section{EXPOSICIÓN DE LOS RESULTADOS}

\section{Introducción al relato biográfico}

Cataluña fue de las últimas regiones de España en sufrir los efectos directos de la GCE sobre el territorio, sin embargo sus consecuencias se hicieron sentir desde las primeras semanas. La asistencia sanitaria de retaguardia a los soldados heridos y la atención a los refugiados fue constante. La perdida de vidas humanas y las persecuciones de carácter religioso, político o cultural, sembraron de miedo a ciudadanos del poniente catalán. Con la guerra en el territorio, aumentaron los dispositivos asistenciales y con ellos la necesidad de personas encargadas de atender a la población herida y enferma. Muchas mujeres deseando ser útiles en aquella guerra se ofrecieron como enfermeras voluntarias.

Cándida Sala Llanes, (10 noviembre 1918 - 18 diciembre 2011) fue una de estas voluntariosas mujeres. Cándida nació en Tremp, un pueblo del prepirineo de Lleida. Desde muy joven manifestó un espíritu curioso, inquieto y activo, alternaba las tareas de la escuela con la biblioteca y los ensayos de teatro en la sede de Acción Católica. Las primeras experiencias de la GCE en persecución religiosa, la conmovieron profundamente. El miedo y la inseguridad se apoderaron de ella y su familia. En abril de 1938 con la llegada de los nacionales en el pueblo decidió implicarse en la guerra colaborando como enfermera voluntaria. Cuidar de los demás le era familiar, la enfermedad de su hermana pequeña, afectada de meningitis, $y$ la humanidad con la que sus padres la atendían contribuyeron en su motivación por ser enfermera. Por sus 9 meses de servicio como enfermera auxiliar en el hospital de campaña

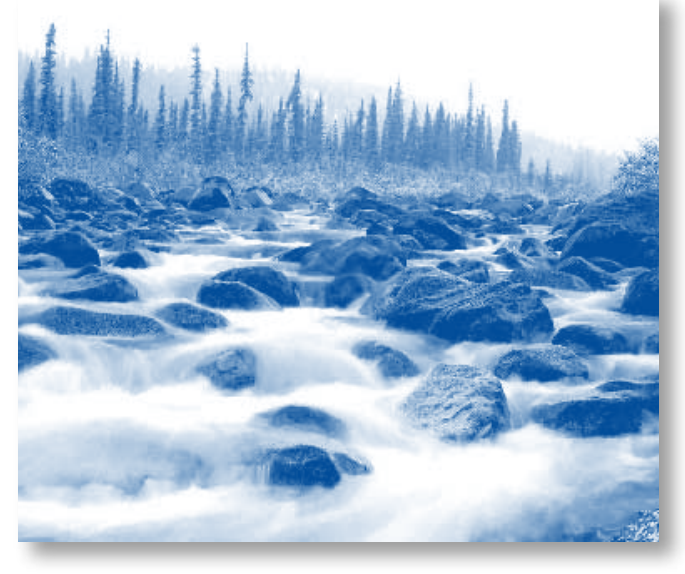

del Cuerpo del Ejército de Urgell Clínica no 1 , fue distinguida con la Medalla de la Campaña.

Acabada la guerra se graduó como enfermera profesional en la Escuela de la Cruz Roja de Barcelona. Ostenta con orgullo su identidad de enfermera de la Cruz Roja. Su actuación en el primer intercambio de heridos y prisioneros de guerra (alemanes e ingleses) de la II Guerra Mundial, llevada a cabo en el puerto de Barcelona en 1943, fue reconocida y elogiada por los embajadores de España en Inglaterra y Alemania y por la Condesa de Lacambra, Presidenta de la Junta del Hospital de Barcelona.

Durante los seis años que ejerció en Barcelona acaudaló una variada experiencia profesional como enfermera e instrumentista de cirugía neurovascular en diversos hospitales de la ciudad. Su inquietud y avidez de nuevas experiencias profesionales y la amistad que mantenía con Mercedes Milá le permitieron desplazarse a Bélgica a trabajar como enfermera en el Hospital Edith Cavell. Allí conoció al que seria su marido.

Para esta mujer, como muchas otras de su tiempo, educada de modo prioritario para el matrimonio y los hijos, el trabajo de enfermera fue quedando relegado a un segundo plano de su vida social, hasta dejarlo del todo, en beneficio del cuidado de su familia. 
Los recuerdos de 15 años de experiencia como enfermera, en una de las épocas más convulsas de Europa, se mantienen muy vivos en la mente de Cándida. Nos los ha ofrecido como un regalo, con una mezcla de cariño y de una profunda emoción, que ni las lágrimas que le brotan muy a menudo la detienen.

A partir de los temas identificados en el texto biográfico, como resultado del análisis se han destacado las siguientes categorías y subcategorías.

Primeros contactos con la GC: acercándonos a las vivencias, experiencias y percepciones

- Violencia

- Miedo

- Creencias religiosas

- Sin olvido

- Ideología política

Implicación en la GC: forjando un carácter

- Motivación por compromiso social

- Acción voluntaria como expresión de altruismo

- Atención en la igualdad

- Creencias - Promesa

- Experiencias que marcan

Postguerra: caminos hacia la profesionalización enfermera

- Adaptación al nuevo contexto

- Inquietudes formativas

- Experiencias que impactan: hambre

Postguerra: estudios de enfermera

- Aprendiendo Enfermería

- Implicaciones personales

Inquietudes profesionales en acción

- Consolidando experiencia profesional

- Practica profesional en el extranjero

\section{RELATO}

El relato se desarrolla a partir de las categorías y subcategorías analizadas.

Primeros contactos con la Guerra Civil: acercándonos a las vivencias, experiencias y percepciones.

Tremp tenía 5.000 habitantes y todos nos conocíamos. Los jóvenes nos divertíamos mucho en el pueblo, éramos jóvenes muy sanos. Las familias católicas disponíamos del Casal Catòlic Cultural, un lugar de encuentro con tenis, frontón, gimnasio, biblioteca y sala de cine con películas para todas las edades. Con la guerra se hicieron amos del pueblo los anarquistas de la FAI "como fieras" y llevaron a cabo una terrible persecución religiosa, asesinando a sacerdotes, intelectuales y católicos. Nosotros, mi familia, teníamos miedo porque éramos de Acción Católica y vivíamos asustados de lo que pasaba. Iban a buscar a personas que no habían hecho nada más que ir a misa y comulgar cada día, era horroroso. Mataron al notario que era soltero, no se podía casar porque tenía a su cargo tres mujeres en casa, su madre y dos hermanas, recuerdo que tenía una biblioteca fantástica. A mosén Cinto, un sacerdote muy mayor que casi no se aguantaba y que vivía en casa de una sobrina que lo cuidaba, se lo llevaron y se les murió. Es inhumano, un pueblo donde todos se conocen, fue terrible. Otros se salvaron... uno de ellos, el que más tarde fue vicario general, le hicieron un proceso y pidió defenderse él mismo "quiero defenderme yo" dijo.

Esto no se puede olvidar nunca... Cuando terminó la guerra me dije "da asco este pueblo y me fui a estudiar a la Cruz Roja de Barcelona". Suerte que tuvimos a Franco, no me da vergüenza decirlo, sino Europa hoy sería Rusia... y seríamos todos rusos. 
Implicación en la Guerra Civil: forjando un carácter

Me ofrecí como enfermera voluntaria en el hospital de sangre, cerca del frente del Segre, que improvisaron los nacionales en el Colegio de Religiosas de la Enseñanza de María Inmaculada de Tremp donde yo había estudiado. Eso fue cuando el pueblo fue liberado de los rojos por Franco, en abril de 1938. Decidí presentarme de enfermera porque en casa teníamos una enferma crónica, María, una hermana que a los seis años tuvo una meningitis y no pudieron hacer nada por ella. Sin embargo su cuerpo era sanísimo, vivió hasta los cuarenta y tres años, fíjese, era más alta que yo. Imagine lo que supone esto para una familia. De mis padres aprendí la humanidad con que se ocupaban de ella. Yo me dije: "Si María continúa viviendo, yo me haré enfermera y así tendré más facilidad para saber como cuidarla".

Yo era la más joven de las voluntarias en el hospital, me llamaban "la peque". De mi pandilla de amigas había otras voluntarias, Milagros Benet, Pilarín Roses y Pilar Ros Benet. Aprendí pronto a ser enfermera, me fue muy fácil cuidar enfermos viendo como lo hacían las enfermeras, los médicos, practicantes y monjas del formidable equipo sanitario militar. Algunas enfermeras eran de la aristocracia. El equipo llevaba de todo, cocineros, de todo... Atendíamos a soldados rasos, oficiales y a prisioneros, pero a estos últimos no nos era permitido hablarles porque podían ser espías, de hecho, hubo más de un caso. Un día vino de visita al hospital un soldado con la capa y las astas de falangista, se detuvo frente la cama de un enfermo de pulmonía y dijo "este tío es un espía", y sí que lo era, lo descubrió por el color de las espuelas de las botas.

Trabajaba por la tarde hasta las 9 de la noche. La religiosa de las Hijas de Caridad de San
Vicente de Paúl con la que compartí tareas era una excelente profesional y muy buena, los enfermos la abrazaban de tanto que la querían.

Me resulta imposible calcular el número de enfermos que había en el hospital, cambiaban muy a menudo, los más graves, con heridas importantes los trasladaban a Zaragoza, los demás se trataban allí.

Padecían de paludismo, sarna y otras enfermedades corrientes. El paludismo lo provocaba una zona entre los dos frentes (frente del río Segre) que estaba llena de mosquitos. Los dos bandos no se fiaban los unos de los otros, ni se ponían de acuerdo para poner solución al problema de los mosquitos, arrasando con fuego el lugar. El paludismo les daba mucha, mucha fiebre. En cuanto a la sarna, había mucha y era muy contagiosa, con un cepillo se fregaba la piel erosionada, se lavaba bien con agua y les poníamos pomada, al final el tratamiento ya se lo podían hacer solos en el frente, porque sino no quedaba espacio en el hospital para otros enfermos. Los cuidados estaban impregnados de una fuerte humanidad, lo importante para nosotras, las enfermeras, era la persona y su bienestar.

En cuanto a los tratamientos farmacológicos, principalmente eran a base de inyecciones para evitar las gangrenas, pomadas, agua oxigenada, yodo y alcohol. Para curar a los soldados de pulmonía les aplicábamos cataplasmas con mostaza, les daba mucho calor y a menudo me pedían "sáquemelo ya" "que cruel que es usted" y yo les contestaba "ahora me dices esto, pero después me lo agradecerás". Y así sucedía, una vez estaban recuperados de la afección pulmonar, volvían para verme y darme las gracias de contentos que estaban conmigo.

Había muchos voluntarios del País Vasco, eran muy majos, conocí a 3 hermanos, el menor de ellos con 12 años. El vasco es una 
persona muy cuadrada pero muy fantástica. También tuvimos legionarios de la XIII Bandera de la Legión, pobres legionarios, el pueblo se encariñaba con ellos, con gran humanidad, les tricotaban calcetines, jerseys, les lavaban la ropa que estaba llena de piojos, les ayudaban en todo y les hacían sentir como si fueran de la familia. Los enviaron a la Batalla del Ebro, y atravesaron el río Ebro para liberar Belchite. Se despidieron del pueblo diciendo, "si nos dejan vivos volveremos", pero no regresó ninguno, los mataron como moscas, todos murieron.

Franco montó comedores en una de las casas del pueblo para dar de comer a la población y atender a los niños. Algunas jóvenes voluntarias ayudaban y de esta forma obtenían el Servicio Social. Creo que es positivo que las chicas hagan el Servicio Social, hoy deberían hacerlo también, porque se casan y no saben como manejar una criatura. Yo recuerdo que no lo quise hacer, había hecho de voluntaria en la guerra como un soldado, había hecho los tres años de estudios de enfermera sin cobrar un duro, nos lo teníamos que pagar todo, los uniformes, los cuellos, todo... y me dije, "yo no haré el Servicio Social”, y pagué cara mi terquedad. Cuando quise salir de España para trabajar necesité el pasaporte y para sacármelo hacía falta el Servicio Social y yo no lo tenía, suerte que en la Cruz Roja encontré personas que me ayudaron. Me dirigieron al jefe de policía, yo no tenía miedo de nada, me dio un abrazo y me dijo "ven a las 12 a buscar el pasaporte". Al recogerlo me dijo "que tengas suerte".

En mi casa de Tremp tuvimos hospedado a un general en las habitaciones que teníamos disponibles. Otro general, Muñoz Grande, estuvo también en Tremp, era majísimo, sencillo, no llevaba nunca la estrella, se mezclaba con los soldados, y hasta comprobaba el rancho. Era gente fantástica. Los soldados salían cada día a pasear y coqueteaban con las chicas del pueblo.

Viví el tremendo caso de un legionario de la XIII Bandera de la Legión que me impactó fuertemente, estaba enamorado de una chica que no era de su clase, cuyos padres no les permitían casarse. Tenía metralla en las piernas, no se podía curar, el médico y las enfermeras de la sala estábamos pendientes de él porque era un santo de bueno, sin embargo el médico nos dijo que no lo aguantaría. El joven pidió a un amigo que se pusiera en contacto con la chica: "escríbele y dile [llorando de emoción] que muero pensando en ella; que la he querido siempre y he sido feliz".

Posguerra: caminos hacia la profesionalización enfermera

La gente después de la Guerra Civil quedó muy triste en Tremp, muchas personas habían perdido algún familiar. Yo ya no fui más al colegio, ya era demasiado mayor, en cambio era muy inquieta, siempre hacía alguna cosa. Aprendí corte y confección y me hacía unos vestidos muy bonitos. Ya a los 17 años mi madre me dijo, "si no te haces tu los jerseys, yo ya he terminado de hacértelos". Aprendí pronto a tricotar y me hacía unos jerseys preciosos. No había otra en el pueblo que los hiciera mejor que yo. También conseguí una máquina de escribir y aprendí mecanografía con sólo dos dedos.

Con 22 años me fui a Barcelona a estudiar de enfermera a la Cruz Roja. No había estado nunca en Barcelona, pero me la aprendí toda, porqué tenía que salir y andar mucho. Lo peor fue el metro, me daba mucho miedo bajar al metro, por lo demás, me lo pasé muy bien, muy bien.

La guerra me impresionó mucho y cuándo finalizó, ya en la Cruz Roja de Barcelona, 
lo que más me impactó fue el hambre. Nos daban arroz hervido, si llegabas de las primeras al comedor te lo podías comer, sino lo tirabas contra la pared y se quedaba pegado. Arroz a mediodía y judías por la noche. Mi familia de Tremp me enviaba paquetes y a mi compañera de habitación, Carmen Catalá Lloret, tía de la actual presidenta del Parlamento de Cataluña, Núria de Gisbert, también le mandaban. Yo a veces me iba a comer en la ducha, porque no tenía para dar a todas. Igual hacía para comer los botecitos de arroz con leche que me preparaba una chica del servicio. Un día, un señor muy mayor, que conocí en la Cruz Roja, me llevó a cenar al mejor restaurante de la Barceloneta.

La posguerra fue muy dura. En Europa, en 1948 implantaron el plan Marshal, una organización de ayuda de los Estados Unidos de América para la reconstrucción de los países europeos después de la Segunda Guerra Mundial. A España, como Franco era amigo de Hitler, no querían ayudarnos los americanos, fueron unos "cochinos", perdone la expresión. Franco fue muy listo. Hitler quería que España entrase en la guerra y él "le hizo la silla", pero nunca entró en la guerra, imagínese si hubiésemos entrado en guerra... Gibraltar sería... nazi. Mi familia, con mi hermana enferma que comía todo distinto, le hacíamos huevos triturados, con pan, con azúcar, cremas, flanes. El azúcar nos lo daban racionado. No podíamos esperar ayuda de nadie... En cuanto al hambre, se pasó peor en la posguerra que en la guerra.

\section{Posguerra: estudios de enfermera}

Trabajábamos 12 horas cada día entre el estudio y las prácticas. Y cuando nos tocaba turno de noche también hacíamos 12 horas. Una tenía 3 dispensarios, otra le tocaba 2 salas generales: una de hombres y una de mujeres,

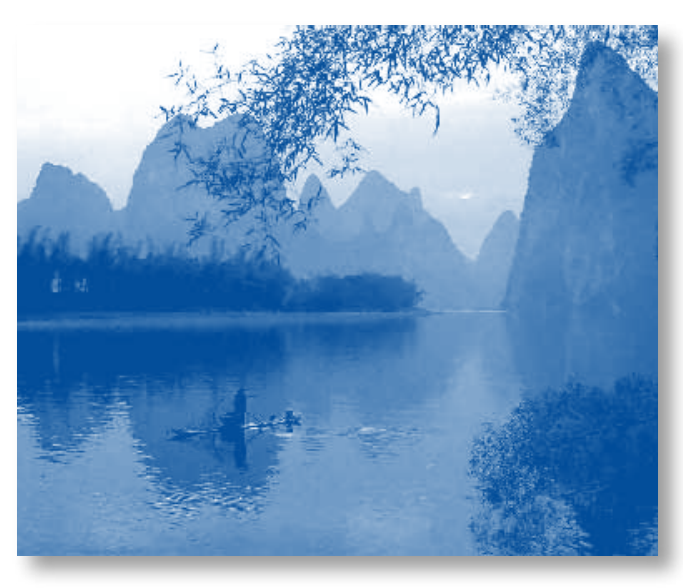

y había 21 enfermos en cada sala. Solas pasamos mucho miedo y muchas calamidades. Las monjas, déjalas correr, querían las cosas bien hechas, esto es de agradecer..., pero teníamos muchas horas de prácticas. Yo me lo pasé muy bien con los enfermos.

Hice muchas amigas en la Cruz Roja, una de ellas Amparo Pallarés, juntas en la escuela estudiábamos y yo decía: "primero quiero terminar la carrera, si me tengo que quedar un año más no me importa", cómo me gustaba mucho el ambiente de la escuela me daba igual. Las matrículas no me interesaban, lo importante para mi era acabar la carrera.

Íbamos siempre de uniforme, el primer año no llevábamos cofia sino que llevábamos sombrero y en verano en el tranvía con las ventanas abiertas, el sombrero volaba y salía despedido por la ventana, y decíamos al conductor "pare, pare" y todo el mundo en el tranvía reía, era divertidísimo. Las enfermeras de la Cruz Roja éramos famosas en el barrio. No nos dejaban salir nunca, pero a la verbena del Poble Nou sí fuimos y reímos mucho.

Participé como enfermera en el primer intercambio de heridos y prisioneros de guerra (alemanes e ingleses) que se hizo durante la Segunda Guerra Mundial en el puerto de Barcelona, el 27 de octubre de 1943. De las 8 de la mañana a la 8 de la noche, ocupándonos de 
ellos, ninguno hablaba catalán. Algunos estaban heridos, llevaban muletas, otros estaban muy sanos, había de todo. Nosotros les dábamos pesetas y ellos nos daban dólares, porque querían bajar al puerto a beber cerveza. Nos lo pasamos muy bien. Fue muy agradable. Lo filmaron todo, y yo salgo en el NO-DO, yo lo tengo... no hay que perderlo porque le costó mucho a mi hijo conseguir una copia.

Después de tres años de estudios, en 1946 y en un ambiente de disciplina militar, obtuve mi título de enfermera de la Cruz Roja. También tengo el titulo por la Facultad de Medicina de Barcelona, porque hubo un momento que el título de la Cruz Roja no era oficial. El hecho de ser voluntaria de guerra no me valió para nada en mis estudios, no me acortaron los estudios.

\section{Inquietudes profesionales en acción}

Al finalizar los estudios trabajé de enfermera en el Instituto Neurológico Municipal de Barcelona hasta 1952. Entré a formar parte como instrumentista del equipo del doctor Antonio Rodríguez Arias, médico neurovascular, acompañándolo en las diversas intervenciones que efectuaba en el Hospital Clínico, el Hospital de la Santa Cruz y San Pablo, el Instituto Neurológico y la Clínica Corachán. Durante un tiempo trabajé en el Sanatorio antituberculoso del Montseny, y en el Dispensario de Angiología del doctor Joan Gibert-Queraltó.

¿Quiere saber como me fui a Bélgica? Yo era muy aventurera. Conocí a Mercedes Milá a través de Concha, una prima suya de Barcelona que trabajaba de telefonista en la Clínica. Ellas me facilitaron los papeles y los contactos para salir de España y encontrar trabajo. Me fui acompañada de Teresa Rull, una compañera que había sido secretaria del Presidente de la Cruz Roja, el doctor García Tornel. El título de enfermera que me valió en Bélgica fue el de la Cruz Roja. Trabajé en el Instituto Edith Cavell - Marie Depage un gran centro hospitalario donde había Escuela de Enfermeras, de Quiroprácticos y otras más... Estuve 5 meses estudiando francés, no sabía nada del idioma. Aprendí muy deprisa porque nadie te hablaba español. Primero trabajé en un servicio de pacientes adultos de muchas nacionalidades, americanos, etc. Allí conocí al que seria mi marido. Los enfermos estaban pocos días, cambiaban a menudo. Después estuve trabajando en la "pouponière", en pediatría, con bebés en incubadoras, en este servicio estuve 5 meses. Me sirvió de mucho trabajar con bebés, cuando me casé y tuve a mis dos hijos, ya sabía hacer de todo, la ropa, los biberones, todo. En Bélgica, en aquel tiempo, cuando nacía una criatura en una familia, una asistencia social pasaba cada mes para comprobar como se encontraba la madre y su hijo.

Desde 1970 vivo en Lleida. Me quedé viuda en octubre de 2009. No he vuelto más a Tremp, tengo recuerdos demasiado tristes, tengo muchas heridas abiertas. Todas mis amigas han muerto. Teresa Rull, de Bélgica se fue al Canadá, nos escribíamos y nos felicitábamos cada año hasta que un buen día devolvieron la carta que yo le había enviado.

\section{DISCUSIÓN}

Interpretando los resultados del análisis vemos que lo que impera en este relato es el espíritu de rebeldía, compromiso, promoción personal y profesional y de aventura de una joven enfermera adelantada a su tiempo. La experiencia de una adolescencia serena y feliz, en un ambiente rural, es el punto de partida de su relato de vida. Una adolescencia truncada brutalmente por la guerra y sus consecuencias. La violencia, persecución y muerte por expre- 
sar la fe católica, hacen temer su seguridad y la de los suyos. Su formación intelectual, las actividades culturales y las prácticas religiosas de la joven son desplazadas por el miedo. En la GCE en Cataluña, ambos bandos enfrentados, el republicano y el nacional llevaron a cabo acciones represivas de tipo político, sindical, religioso o cultural sobre determinadas personas o grupos de población. La libertad, y la justicia, para Cándida y su familia, fueron restituidas por los nacionales al ganar la guerra. Su verdad de la historia de la GCE, es tan verdadera, como verdaderas son las verdades de las historias de personas y familias que se vieron privadas de libertad y justicia al perderla.

La rebeldía se refleja en el acento crítico de su discurso al referirse a los acontecimientos adversos que le toco vivir. Aún hoy, después de tantos años, dice "no se puede olvidar nunca" manteniendo un profundo resentimiento y defendiendo su postura anticomunista.

El compromiso social se manifiesta al querer ser útil en aquella guerra como voluntaria para ayudar a los demás. Nada ni nadie es más fuerte que una voluntaria. Es el mismo tipo de ejemplo extraordinario de entrega y compromiso que demostraron sus contemporáneas en sus relatos sobre las experiencias de guerra como enfermeras voluntarias (Via, 2006); (Valls, 2008). Un compromiso a la vez motivado por la autopromesa de estudiar enfermera con el fin de ofrecer a su hermana enferma los mejores cuidados de enfermería. Cuidó de los soldados nacionales y de los prisioneros republicanos, era frecuente que estuvieran mezclados unos y otros en la misma dependencia (Jakson, 2004), como otras enfermeras de uno y otro bando, no hacía distinciones partidistas a la hora de cuidar (Amezcua, 1996), su deber humanitario y profesional era velar por el bienestar de todos. Tuvo una buena maestra en su compañera de trabajo, la religiosa de San Vicente de Paúl, de todos es bien conocido el reconocimiento social y profesional de esta Congregación por sus aportaciones a la Enfermería (Hernández, 2006) y a la sanidad. Otras congregaciones de religiosas mantuvieron un destacado papel como enfermeras durante el conflicto bélico en ambos bandos (Linage, 1999). Los cuidados y tratamientos que dispensaba Cándida coinciden con los relatados por las demás enfermeras de guerra catalanas (Valls, 2008), cuidados a los heridos y enfermos de afecciones infecciosas causadas por la sarna y aplicación de tratamientos a base de inyectables, pomadas y desinfectantes. Lo más destacado del cuidado era el aspecto humanitario del mismo y la gratitud con que respondían los enfermos y heridos tratados por ella.

La promoción personal la acompaña a lo largo de su vida imprimiendo en ella un fuerte carácter de esfuerzo de superación personal y estímulo profesional que al desarrollarse al amparo del bando vencedor no tuvo grandes impedimentos en canalizar sus aspiraciones profesionales, contrariamente a las enfermeras del bando republicano que encontraron muchas dificultades o les fue imposible formarse para devenir enfermeras profesionales (Torres, 2007). Sin duda, su implicación personal como enfermera contribuyo hacer visible, el papel de la mujer en la GCE como una forma de participación política (Díaz, 2005). Su testimonio confirma, una vez más, que en la GCE la enfermería fue uno de los ámbitos más importantes de movilización femenina (Nash, 1999) y de promoción de nuevas profesionales, entre las mujeres del ámbito rural y pertenecientes a sectores sociales populares (Larraz, 2005).

Su tesón en cumplir la promesa de estudiar enfermera le permitió sobrellevar el hambre de la postguerra. El mismo tesón demostró en 
acabar la carrera, "las matriculas no me interesaban" afirma, "lo importante era acabar la carrera" de enfermera de Cruz Roja en aquel ambiente de disciplina militar. Los tiempos de guerra y de hambre fueron los que más le impactaron de adolescente y joven estudiante.

$\mathrm{Su}$ valía profesional la demuestra siendo enfermera en los más prestigiosos centros sanitarios y equipos de Barcelona. Su espíritu inquieto y aventurero, la llevan más tarde a probar suerte y afianzar su experiencia profesional en el Hospital Edith Cavell de Bélgica, en un tiempo, donde sólo las más comprometidas oficialmente con la profesión enfermera, como su amiga Mercedes Milá, podían salir de España.

En su relato no hay olvido sino memoria. Es la memoria de la más joven de las voluntarias de guerra de su hospital comprometida con el cuidado de los que la necesitaban. Es la memoria de las vicisitudes por formarse profesionalmente. La memoria del hambre. La memoria de sus logros profesionales y familiares. Es también la memoria de "demasiados recuerdos tristes,.... y de muchas heridas abiertas" de la GCE, la que le impidió de mayor regresar al Tremp de su adolescencia.

Sus metas, ser enfermera, esposa y madre las alcanza satisfactoriamente.

Han pasado casi dos décadas desde que uno de los pioneros en investigación histórica de la enfermería en nuestro país, animaba a los historiadores de la enfermería de GCE a seguir trabajando a partir de fuentes escritas. Sugería, a la vez, tomar conciencia de la urgencia de la investigación oral a través de las historias de vida de enfermeras de guerra que sobrevivieron al conflicto... "los documentos pueden aguardar... ellas no" (Siles, 1996). Pasados tantos años de la GCE, aún es posible para los investigadores de la enfermería encontrar enfermeras como Cándida que pueden darnos de viva voz su testimonio.

\section{CONCLUSIONES}

Después de 75 años de la GCE, ha sido posible sumar una nueva historia de la contribución enfermera en la GCE, desde la mirada de una joven voluntaria en un hospital de sangre del bando nacional, cerca del frente del Segre, en el territorio catalán.

Su motivación por implicarse en el conflicto fue por compromiso social y familiar como persona, y por aspirar a una formación profesional y promoción personal como mujer. Los cuidados que dispensaba estaban impregnados de un fuerte carácter humanitario, recibiendo a cambio gratitud de sus pacientes.

Aplicaba tratamientos curativos a enfermos de pulmonía, sarna y paludismo y tratamientos preventivos contra las infecciones y gangrenas. Fue distinguida por sus servicios de enfermera voluntaria de guerra con la $\mathrm{Me}$ dalla de la Campaña.

Su vivencia de la guerra y la del hambre de la posguerra, mientras estudiaba enfermería en la Cruz Roja de Barcelona, la impactaron profundamente.

Desarrolló su experiencia profesional de enfermera en diversos centros y servicios de Barcelona y en el hospital Edith Cavell de Bruselas.

A pesar del contexto en que vivió sobresale por sus cualidades de mujer fuerte, comprometida con la sociedad y su familia, de espíritu rebelde y aventurero, que entendió bien su papel profesional y asumió sus responsabilidades. Visualizar su experiencia, en una época difícil especialmente para las mujeres, nos ayuda a reflexionar sobre la asunción de nuestro compromiso social y profesional en el marco de la actual crisis económica que repercute en el sistema sanitario y en nuestra profesión. 


\section{BIBLIOGRAFÍA}

- Amezcua, M. (1996) Enfermeras en La Guerra Civil Española. Impresiones y testimonios. Índex de Enfermería (15), 9-10

- Amezcua, M., Hueso Montoro, C. (2006) Cómo elaborar un relato biográfico. Archivos de la Memória 1. Disponible en: http://www.index-f.com/memoria/metodologia. php. Consultado 15/02/12.

- Amezcua, M. Hueso Montoro, C. (2009) Cómo analizar un relato biográfico. Archivos de la Memória 6 . Disponible en: http://www.index-f.com/memoria/6/ mc30863r.php. Consultado 6/02/12.

- Hernández, F. (2006) Las Hijas de la Caridad en la profesionalización de la Enfermería. Cultura de los Cuidados (20), 39-49.

- Jackson, A. (2004) Més enllà del camp de batalla. Testimoni, memòria i record d'una cova hospital en la Guerra Civil espanyola. Cossetània, Valls.

- Larraz, A., Ibarrola, C., Pardo, M. (2005) El Hospital Alfonso Carlos de Pamplona (1936-1939): un hito en la promoción de la mujer y el desarrollo de la enfermería en Navarra. Temperamentum 2. Disponible en: http:// www.index-f.com/temperamentum/tn2/t0125r.php. Consultado 14/02/12.
- Linage, A. (1999) En torno a las enfermeras de la Guerra Civil. Híades (5-6), 169-187.

- Lincoln, Y.S. Guba, E.G. (1985)Naturalistic Inquiry. Sage Beverly Hills.

- Nash, M. (1999) Las mujeres republicanas en la guerra civil. Taurus, Madrid.

- Pujades, JJ. (1992) El Método biográfico: El uso de las historias de vida en ciencias sociales. Elaboración de una historia de vida. (2a ed.), Centro de Investigaciones Sociológicas, Madrid, 59-84.

- Siles, J. (1996) Enfermería y conflictos bélicos. Una historia por hacer. Index de Enfermería (15), 7-8.

- Torres, C., Valls, R., Ramió, A., Domingo, A., Rodero, V., Vives, C. (2007) Las enfermeras catalanas en la Guerra Civil española. Temperamentum 6. Disponible en: http://www.index-f.com/temperamentum/tn6/t2707r. php. Consultado 9/02/12.

- Valls, R., Ramió, A., Torres, C., Roldán, A., Rodero, V., Vives, C. (2007) Infermeres catalanes a la Guerra Civil. Publicacions i Edicions de la Universitat de Barcelona, Barcelona.

- Via, R. (2006) Nit de Reis: diari d'una infermera de 14 anys (del setembre de 1938 al juny de 1939). Edicions $i$ Propostes Culturals, Vilafranca del Penedès. 